\title{
The potential anti- African Swine Fever Virus effects of medium chain fatty acids on in vitro pig feed and water models
}

Ha Thi Thanh Tran

National Institute of Veterinary Research

Anh Duc Truong

National Institute of Veterinary Research

Duc Viet Ly

National Institute of Veterinary Research

Tuan Van Hoang

National Institute of Veterinary Research

Chinh Thi Nguyen

National Institute of Veterinary Research

Nhu Thi Chu

National Institute of Veterinary Research

Huyen Thi Nguyen

National Institute of Veterinary Research

Anh Thi Kieu Dang

National Institute of Veterinary Research

Maartje De Vos

RAG

Kobe Lannoo

Evonik Industries AG

Geert Bruggeman

RAG Aktiengesellschaft

Hoang Vu Dang ( $\nabla$ dangnivr@yahoo.com )

National Institute of Veterinary Research

Research article

Keywords: African swine fever virus, virus isolation, MCFA, pig, Vietnam

Posted Date: June 4th, 2020

DOI: https://doi.org/10.21203/rs.3.rs-27996/v1 
License: (c) (i) This work is licensed under a Creative Commons Attribution 4.0 International License. Read Full License 


\section{Abstract \\ Background}

African swine fever (ASF) is an important disease affecting swine and has a significant economic loss on both the developed and developing world but due to the lack of vaccines, drug, and effective control measures, ASF virus remains a serious threat to global pork production. The activities of medium-chain fatty acids (MCFAs) against viral pathogens have been reported previously. However, the effects of this family on ASFV have been not yet investigated. In this study, we evaluated the potential effects of MCFAs in individual and synergistic forms, to prevent and/or reduce ASFV infection using in vitro feed and water models.

\section{Results}

The potential effects of MCFAs, including C8, C6-C8-C10 (1:1:1 ratio) and C8-C10-C12 (1:1:1 ratio) against a field ASFV strain isolated in Red River Delta region of Vietnam were further examined by real-time PCR in in vitro feed and water models. All tested products have shown a strong antiviral effect against ASFV infectivity at doses of $0.375 \%$ and $0.5 \%$ in both feed and water assays. Interestingly, the synergistic MCFAs have shown clearly their potential activities against ASFV in which at lower dose of $0.25 \%$, pretreatment with product 2 and 3 induced significant increases at the level of $\mathrm{Cq}$ value when compared to positive control and/or product $1(P<0.05)$.

\section{Conclusions}

To our knowledge, it is the first report on in vitro examination of the anti-ASFV activities of the MCFAs. Our findings suggested that all tested products, both individual and synergistic forms of MCFAs, have possessed a strong anti-ASFV effect and this effect is dose- dependence in in vitro feed and water models. Additionally, synergistic effects of MCFAs are more effective against ASFV when compared to individual form. The further studies focusing on in vivo anti-ASFV effects of MCFAs are very important to bring new insight into the mode of ASFV-reduced action by these compounds in swine feed and water consumption.

\section{Background}

African swine fever (ASF) was first observed in Kenya in 1909 following the introduction of the European (exotic) domestic pig and described retrospectively in 1921 as a highly contagious hemorrhagic disease in both wild boar and domestic pigs [1]. ASF virus (ASFV) has extremely high morbidity, mortality spreads rapidly, and causes severe illnesses up to $100 \%$; thus, it is a notifiable as an important disease with the World Organization for Animal Health (OIE) [2]. On August 2018, ASFV made its biggest leap into China and then outbreaks in Asiatic countries that have highlighted the urgent need for effective antiviral agents 
against ASFV, which can provide an alternative tool for combating this disease [3-6]. Due to the lack of vaccines, drug, and effective control measures, ASFV remains a serious threat to global pork production $[7,8]$. Currently, one of the most effective measures to control and prevent ASFV-spread is biosecurity [9, 10], including biosecurity at farm, local and national levels.

The recent results demonstrated that ASFV could be easily transmitted orally through natural consumption of both liquid and feed, indicating the important role of feed in the emergence of ASFV outbreak in new pig populations throughout the world [11, 12]. Disinfection and the proper use of disinfectants of pathogenic in feed and water are a basic and the most important role in the aspect of biosecurity and based on decreasing the percentage of pathogenic micro-organisms in the feed $[10,13$,

14]. The ideal of "feed and water biosecurity" should be characterized by non-toxicity, fast action, durability, resistance to influence from the environment, and more importantly, it should have the widest possible spectrum of biocidal activity, including bacteria, viruses, and fungi. The incorrect definition of activity parameters such as concentration, contact time, and range may lead to the improper use of disinfectant products, whereby no effectiveness is achieved [11-14]. There are several substances or chemical compounds generally accepted as inactivating enveloped viruses, including the ASFV and the only studies on the use of disinfectants against the ASFV concerning tests on various surfaces, as revealed [15-18]. To the best of our knowledge, this is the first in vitro testing of medium-chain fatty acids (MCFAs) as chemicals/disinfectants against the ASFV in swine feed and water models. Therefore, the aim of this experiment focused on (i) characterization of the ASFV strain isolated from the capital Hanoi outbreak in Red River Delta region of Vietnam and (ii) evaluation of the potential effects of MCFAs, individual and synergistic forms, to prevent or reduce the amounts of viral particles of ASFV in in vitro feed and water models.

\section{Results And Discussion}

African swine fever (ASF) is one of the most important viral diseases of wild boar and domestic pigs, causing significant socio-economic losses in affected countries [19]. Depending on host characteristics and the circulating viral isolate, clinical signs may vary from the highly lethal form with $100 \%$ mortality to sub-clinical and a clinical form [9, 19-21]. Since the developed vaccines against ASFV have been failing to induce effective protection, it is reasonable to evaluate antiviral agents against this virus $[7,8,10]$. The aim of this study focused on $(I)$ characterization of ASFV strain isolated from the capital Hanoi outbreak in Red River Delta region of Vietnam and (ii) investigation of the antiviral activities of MCFAs at different composing materials, including C8 (caprylic acid), C8-C6-C10 (caprylic acid-caproic acid-capric acid) and C8-C10-C12 (caprylic acid-capric acid-lauric acid), against ASFV infection in vitro. We firstly screened the cytotoxicity of MCFAs on PAMs cells, the permissive cells for ASFV infection in vitro, by using the MTT assay according to the manufacturer's protocols. As shown in Fig. 1B, at the dose of $200 \mu \mathrm{g} / \mathrm{ml}$, products 1 and 3 have shown a less cytotoxic effect when compared to product 2 on PAM cells, suggesting the anti-proliferative effects of $\mathrm{C} 6$ component in product 2. All products do not induce the cytotoxicity at the dose of $100 \mu \mathrm{g} / \mathrm{ml}$ (Fig. 1B), suggesting that all products are suitable for further in vitro examination. It has been reported previously that $\mathrm{C} 6$ and $\mathrm{C} 10$ compounds at low concentration may cause an alteration 
in cell membrane structures. However, the cytotoxic effects on mammalian cells may not reflect the adverse effects in humans and animals because of many physiological factors, including mucins and serum [22].

In order to examine antiviral effects of chemical/disinfectants, information of viral strain used in whole experiments is very important, including genetic characterization. In order to evaluate the antiviral effects of MCFAs, an ASFV isolated from an outbreak in the capital Hanoi of Vietnam was employed. Some virus assays, including virus isolation in combination with HAD assay and conventional PCR for genotyping and serotyping have been performed to confirm this strain. In Fig. 2A, a strong positive HAD was observed in culture, indicating the success of viral isolation. This isolate was named as Pig/Hanoi/2019/01. Further conventional PCR was conducted for verification of HAD positive data as recommended by OIE and genetic characterization of Pig/Hanoi/2019/01 based on the sequencing analysis of the $\mathrm{p} 72, \mathrm{CD} 2 \mathrm{v}$, and TRS region gene. As expected, positive 478-bp, 816-bp, and 356-bp bands of PCR products were appeared on gel, respectively (Fig. 2B).

Nucleotide sequence comparisons using the Basic Local Alignment Search Tool (http://blast.ncbi. nlm.nih.gov/Blast.cgi) revealed that the p72 and CD2v sequences of Pig/Hanoi/2019/01 were $100 \%$ identical to those of China isolates of ASFV SY18, Pig/HLJ/2018, AnhuiXCGQ, and CN201801 [3, 23, 24]. Phylogenetic analysis of p72 and CD2v of ASFV strain Pig/Hanoi/2019/01 demonstrated that the ASFV, $\mathrm{Pig} / \mathrm{Hanoi} / 2019 / 01$, belongs to genotype II and serotype 8, and closely related with ASFVs isolated from China in 2018 [3], Russia in 2012 and 2017 [25] and Estonia 2014 [26] (Fig. 3A-B). On the other hand, the TRS regions in the IGR between I73R and I329L has been extremely useful for resolving epidemiologic complexities at the genotype, region, and country levels [27-29], such that additional genome markers are required to evaluate the origin and to map the outbreak of closely related ASF isolates circulating in Viet Nam. Our results showed that the Pig/Hanoi/2019/01 strain had an intergenic region II variant with an additional 10 nucleotide (5'-GGAATATATA-3') into the TRS region between the $173 \mathrm{R}$ and I329L genes (Fig. 3C). The intergenic region II variant of the Pig/Hanoi/2019/01 strain was identified to those described in isolates Ukr12/Zapo, Belgium 2018/1 [27]. The same TRS insertion was also found in China isolates of ASFV SY18, Pig/HLJ/2018, AnhuiXCGQ, and CN201801 [3, 23, 24] and differ from ASFV isolated in Georgia in 2007 [30]. The sequences of p72, CD2v, and TRS genes of Pig/Hanoi/2019/01 strain have been deposited in GenBank as the accession numbers of MT332151-3, respectively.

Recent research suggested that the susceptibility to chemicals/disinfectants depends on viral characteristics in which non-enveloped viruses are more resistant than enveloped viruses [31]. Moreover, some chemical synthesis or extract from a plant such as an acacetin, apigenin, genkwanin, rhoifolin, vitexin, and vitexin 2-0-rhamnoside are able to inhibit or reduced ASF virus-specific protein synthesis and viral factory formation in Vero cell line system, in which apigenin showed potent inhibition of ASF virusinfected Vero cells with not display a cytopathic effect [17, 18, 32]. However, there is no evidence of the survivability of ASFV in feed and water after pre-treatment with MCFAs. On the other hand, a recent report demonstrated that the activity of MCFAs could enhance the RNA degradation and mitigating of PEDV in swine feed and ingredients [16]. To investigate the potent effects of MCFAs to reduce Pig/Hanoi/2019/01 
infectivity, we used both in vitro feed and water models in which MCFA pre-treated feed and water were spiked to Pig/Hanoi/2019/01 isolate at $10^{5}$ and $10^{1}$ HAD50, respectively and the samples were collected after $24 \mathrm{~h}$ post-inoculation. Real-time PCR amplification of the $\mathrm{p} 72$ gene was performed to detect the presence of viral DNA in feed and water based on OIE recommended protocol. A recent in vivo report has indicated that the minimum infectious dose of ASFV in feed is $10^{4} \mathrm{TCID}_{50}$ in which $40 \%$ of pigs orally exposed to ASFV have shown s positive result, while other study suggested that minimum dose of ASFV oral exposure should be $10^{5}$ (Niederwerder et al., 2019). The results of MCFA pre-treated feed exposed to ASFV at the dose of $10^{5} \mathrm{HAD} 50$ are summarized in Fig. 4. No Cq value was obtained in the negative control group, while feed exposed to ASFV without MCFA treatment as a positive control group shown the Cq value ranged from $25.85 \pm 0.67$ to $26.52 \pm 0.82$ after $24 \mathrm{~h}$. It is indicated that the ASFV could survive in the feed consumption and can be spread the ASFV in the new population of the pigs industry [11, 33, 34]. In feed experiment group, a strong anti-ASFV effect was observed in all product treatment after $24 \mathrm{~h}$ postinoculation. Pre- treatment with product 1 significantly reduced ASFV replication at two highest doses of 0.375 and $0.5 \%(P<0.01)$ (Fig. 4). Product 2 has shown the potential susceptibility to ASFV isolated in Vietnam in which all doses $(0.125 \%$ and $0.25 \%$ with $P<0.05 ; 0.375 \%$, and $0.5 \%$ with $P<0.01)$ induced a statistical increase in $\mathrm{Cq}$ value when compared to a positive control. On the other hand, at the dose of $0.25 \%$, product 3 caused significantly enhancement at the levels of the Cq value when compared to a positive control $(P<0.05)$ and a significant increase in dose- dependence was recognized at doses 0.375 and $0.5 \%$ in product 3 group $(P<0.01)$ (Fig. 4$)$. These results demonstrated that all products of MCFAs significantly increased in the Cq value when compared to the positive control $(P<0.01)$ at the highest doses of $0.375 \%$ and $0.5 \%$ at $24 \mathrm{~h}$ after virus incubation (Fig. 4). Additionally, our findings also indicated that MCFAs in synergistic forms have shown promising candidates to reduce ASFV infectivity in which product 2 reduced ASFV infectivity at the lowest dose of $0.125 \%$, while a significant increase in Cq value was noted in product 3 group at a dose of $0.25 \%(P<0.05)$, suggesting that synergistic MCFA C6-C8-C10 is the most potential MCFAs against ASFV in feed model. It is clear that the antiviral effects of MCFAs on the reduction of ASFV infectivity in feed depends on the composing materials in the mixture and the dose. Recent study in the effects of MCFAs on PEDV infectivity has indicated that C8 component has shown the strongest antiviral effect as an individual component and MCFA blend (C8-C6-C10) is promising product to reduce PEDV in feed. An agreement between our data on ASFV and previous study on PEDV in feed was recognized in this study.

One of the most important steps when performing "feed and water biosecurity" is disinfection, and it may reduce or inhibit the risk of the ASFV contamination in the environment or other pathogens [31]. Recent report demonstrated that the ASFV could be directly transmitted to contract pigs by contaminated fomites, drinking water, facilitate pig industry [12], excretions [13], and carriers [14]. The minimum infectious doses of ASFV for water via the oronasal route was estimated to be $10^{0} \mathrm{TCID}{ }_{50}$, respectively, suggesting that the small amount of infective material could lead to ASFV transmission to the healthy pig industry [11]. To further evaluation of MCFA- activities to reduce ASFV infectivity in swine water consumption, the effect of MCFAs at dose- and component- dependence were also tested. These results shown in Fig. 5 indicated that all products have shown a strong antiviral effect against ASFV infectivity 
at doses of $0.375 \%$ and $0.5 \%(P<0.05)$ in water assays. At the dose of $0.25 \%$, pre-treatment with product 2 and 3 induced significant increases at the level of Cq value, while at lowest dose of product $3(0.125 \%)$, a statistical enhancement was noted after $24 \mathrm{~h}$ post incubation, suggesting that product 3 (C8-C10-C12) is most potential candidate as MCFA additives to reduce ASFV contamination in water model at the time point of $24 \mathrm{~h}$. Further studies in time dependence should be performed to obtain the best understanding of MCFA blend activities against ASFV in swine feed and water consumption. Interestingly, a good correlative result obtained in both in vitro feed and water experiments was observed in which MCFAs, including product 1 , product 2 , and product 3 at doses of $0.375 \%$ and $0.5 \%$ have reduced markedly the survival of ASFV after 24-hour post-inoculation at ASFV titration of $10^{5} \mathrm{HAD} 50$ and $10^{1} \mathrm{HAD} 50$, respectively and MCFAs in synergistic forms are most promising candidates to reduce ASFV infectivity in swine feed and water consumption at the dose of $0.25 \%$. Additionally, the potential effects of MCFAs, individual and synergistic forms, on ASFV infectivity are dose- and component- dependence. It has been indicated that, MCFAs consists of four chemical structures, including caproic acid (C6), caprylic acid (C8), capric acid (C10) and lauric acid (C12). The antimicrobial activities of MCFA have been reported previously. Some studies have suggested the potential antiviral effects of caprylic acid (C8) and/or capric acid (C10) on vesicular stomatitis virus or visa virus [22]. Additionally, the synergistic effects of MCFAs have shown more effective against pathogens when compared to their individual form in which combined effects of $\mathrm{C} 8$ and $\mathrm{C} 10$ caused a significant decline of bacterial flora, while no efficacy was found by individual $\mathrm{C} 8$ or $\mathrm{C} 10$ [35]. As a shortest member of MCFA family, caproic acid C6 and its antimicrobial effects on bacterial growth have been reported in previous study in which at the dose of $25 \mu \mathrm{g} / \mathrm{mL}$ this acid has shown a completed inhibition on actinobacillus actinomycetemcomitans growth, a gram-negative bacterium [36]. The significant activities of $\mathrm{C} 6$ in combination with $\mathrm{C} 8$ and $\mathrm{C} 10$ against PEDV in previous study and our current ASFV experiment indicated that, this compound possessed both anti-bacterial and viral properties. Also, other studies have demonstrated that among all MCFAs, C12 component is majority of antibacterial activities [37,38]. The role of C12 component in synergistic MCFAs was markedly noted in our feed and water experiment, suggesting the susceptibility of lauric acid C12 to viral pathogens. Further studies are essential to elucidate the chemical properties and antiviral activities of this compound. The anti-bacterial effects on MCFAs have been reported in both in vitro and in vivo studies, however the study of their effects on viral pathogens are limited. Although, it is difficult to be definitive regarding to molecular mechanism underlying MCFA, both individual and synergistic forms, activities against ASFV infectivity, it could be investigated in further studies, particularly in vivo model.

\section{Conclusion}

To our knowledge, it is the first report on MCFA- activities against ASFV in in vitro feed and water models. Our findings suggested that the MCFAs possessed the potent activities as an antiviral feed and water mitigant on ASFV infectivity in vitro and the synergistic forms of MCFAs (C6-C8-C10 and/or C8-C10-C12) have shown stronger anti-ASFV activities in vitro when compared to individual form of MCFA (C8). Additionally, the effects of MCFAs on ASFV infectivity are dose- and component- dependences. The 
further studies focusing on in vivo anti- ASFV effects of MCFA are very important to bring new insight into the mode of ASFV-reduced action of these compounds.

\section{Methods}

\section{Virus isolation, DNA extraction, and PCR assay}

Preparation of porcine alveolar macrophages (PAM) cells was done as previously described [4]. The PAMs cells were cultured at $37^{\circ} \mathrm{C}$ in Dulbecco's Modified Eagle Medium (DMEM) supplemented with $10 \%$ FBS, $2 \mathrm{mM}$ L-glutamine, $1000 \mathrm{IU} / \mathrm{ml}$ penicillin, and $1 \mathrm{mg} / \mathrm{ml}$ streptomycin. The homogenate of the field pig spleen sample from ASFV confirmed outbreak in the capital Hanoi was virus-positive by conventional PCR using p72U/p72D specific primer (data not shown), and the haemadsorption (HAD) assay was used to inoculate PAMs for virus isolation as previously described [4]. The cell supernatants were collected after 4 days inoculation and the HAD assay for infectious virus particles was performed. The genomic DNA of ASFV was extracted by using the QIAamp DNA Mini Kit (QIAgen, Hilden, Germany) from cell supernatants. For molecular analysis, different PCRs were set up on ASF isolate: (i) the DNA of ASFV in the supernatant was detected by the real-time PCR for viral p72 gene-specific primer as recommended by OIE, and the real-time PCR was carried out on an Agilent AriaMx Real-Time PCR System (Agilent, SC, CA, USA) according to the OIE-recommended procedure described in King et al. [39]; (ii) the C-terminal region of the p72 protein was amplified using primers p72U/D for genotype analysis [28, 29]; (iii) a fragment of the EP402R gene encoding the $\mathrm{CD} 2 \mathrm{v}$ protein using specific primer CD2-2F/2R according to the previously described for serotype analysis [27]; and (iv) a fragment of the tandem repeat sequences (TRS) in the intergenic area between the 173R and I329L genes using specific primer EC01A/ECO1B according to the previously described $[27,28]$ (the original electrophoresis images gel was shown in Additional file 1). Positive amplification control consisted of known DNA of ASFV positive, and negative amplification control consisted of nuclease-free sterile water.

\section{HAD assay}

The titer of ASFV was performed by HAD assay and expressed as $50 \% \mathrm{HAD}$ doses/ml $\left(\mathrm{HAD}_{50}\right)$, as previously described [2, 4]. Briefly, the HAD assay was performed as previously described [40]. Primary PAMs cells were seeded in 96-well plates, and the samples were then added to the plates and titrated in triplicate using $10 \times$ dilutions. The quantity of the ASFV was determined by identification of characteristic rosette formation representing haemadsorption of erythrocytes around infected cells, according to the guideline of OIE [2]. HAD was observed for 4 days and the images were captured with Optika Vision Pro (OPTIKA S.r.I.Via Rigla, Ponteranica (BG) - Italy). The $\mathrm{HAD}_{50}$ was calculated by using the method of Reed and Muench [41].

\section{Sequence analysis of the ASFV isolate}

The correct size of the amplicons of the partial gene p72, EP402R, and TRS products were electrophoresed on a 1.5\% agarose gel against a 100 bp DNA leader marker (Thermo Scientific) and 
visualized by UV irradiation and ethidium bromide staining (Sigma-Aldrich, St. Louis, MS, USA). The correct size of the amplicons was isolated from the agarose gel and purified using the QIAQuick gel extraction kit (QIAgen) according to the manufacturer's specifications for sequencing (1st BASE, Selangor, Malaysia). The chromatograms of amplicons and probe binding site sequences were analyzed using BioEdit and DNAstar program (DNASTAR Inc. Madison, WI, USA). The nucleotide identity of the ASFV strain in the capital Hanoi outbreak of Vietnam in comparison with other sequences was performed using the Blast tool at the National Center for Biotechnology Information (NCBI) database and using the information of published sequences. The multiple sequence alignment was performed using the Lasergene software (DNASTAR Inc.). Phylogenetic analyses of nucleotides sequences of partial p72 and CD2v ASFV was constructed using the neighbor-joining method with a bootstrap value of 1,000 in the MEGA7 program [42].

\section{Experimental design of feed assay}

The 22.5 grams of commercial swine feed (formula composition Table 1) samples were treated with 03 products of MCFAs, product 1 (RAG C8), product 2 (RAG C6-C8-C10; 1:1:1 ratio) and product 3 (RAG C8C10-C12; 1:1:1 ratio) (All products provided by Royal Agrifirm Group, Landgoedlaan 20, 7325 AW Apeldoorn, The Netherlands; chemical structures as shown in Fig. 1A) at an application rate of 0,125\%, $0,25 \%, 0,375 \%, 0,5 \%$ and then, spiked with $2.5 \mathrm{ml}$ of DMEM (Gibco, Thermo Scientific) containing $1 \times$ $10^{6} \mathrm{HAD}_{50} / \mathrm{ml}$ of ASFV. An additional 22.5 grams of feed samples were inoculated with phosphatebuffered saline (PBS, Sigma-Aldrich) and used as negative controls, while an additional 22.5 grams of feed samples were inoculated with $1 \times 10^{6} \mathrm{HAD}_{50}$ of ASFV and used as positive controls. The final virus titration is $1 \times 10^{5} \mathrm{HAD}_{50} / \mathrm{g}$ feed. Treatments of each group or control group were collected at 24-hour post-inoculation. The sample was collected for DNA extraction by using the QIAamp DNA Mini Kit (QIAgen, Hilden, Germany) and subject to real-time PCR as described above. 
Table 1

Calculated energy and nutrient content of the commercial feed

Items

Crude protein \%

Calcium, \%

Humidity \% (max)

Non-phytate phosphorus, \% (min-max):

Cellular \% (max)

Lysine \% ( $\mathrm{min})$

Methionine + Cystine \% ( $\mathrm{min})$

Metabolizable energy \% (min)

Ingredients: Cereal, soybean meal, fish oil, corn, broken rice, rice bran, vegetable oil, dicalcium phosphate, amino acids (L-Lysine, DL-Met, L-Threonine, L-Tryptophan), enzyme Bio-zeem TM $(1,000 \mathrm{mg} / \mathrm{kg})$, sodium chloride, sodium bicarbonate, copper sulfate, iron glycinate, phytase, premix vitamins, premix organic minerals

\section{Experimental design of water assay}

The $4.5 \mathrm{ml}$ swine water (negative with ASFV) samples were treated with 03 products of MCFAs at an application rate of $0,125 \%, 0,25 \%, 0,375 \%, 0,5 \%$ and then, spiked with $0.5 \mathrm{ml}$ of DMEM (Gibco, Thermo Scientific) containing $1 \times 10^{2} \mathrm{HAD}_{50} / \mathrm{ml}$ of ASFV. An additional $4.5 \mathrm{ml}$ of water samples were inoculated with PBS (Sigma-Aldrich) and used as negative controls, while an additional $4.5 \mathrm{ml}$ of water samples were inoculated with $0.5 \mathrm{ml}$ of $1 \times 10^{2} \mathrm{HAD}_{50}$ of ASFV and used as positive controls. The final virus titration is $1 \times 10^{1} \mathrm{HAD}_{50} / \mathrm{ml}$ water. Treatments of each group or control group were collected 24-hour post-inoculation. The sample was collected for DNA extraction by using the QIAamp DNA Mini Kit (QIAgen, Hilden, Germany) and subject to real-time PCR as described above.

\section{Cytotoxicity of MCFAs on PAM cells}

PAMs cell was harvested, counted by hemocytometer and diluted with medium, yielding a concentration of $1 \times 10^{6} \mathrm{cells} / \mathrm{ml}$. From this cell suspension, $100 \mu \mathrm{l}$ was pipetted into $96-$ well microtiter plates (Nunc, Denmark) and incubated for 24 hours in a $5 \% \mathrm{CO} 2$ incubator at $37^{\circ} \mathrm{C}$. Cells were then treated with $25 \mu \mathrm{g}$, $50 \mu \mathrm{g}, 100 \mu \mathrm{g}, 200 \mu \mathrm{g}, 400 \mu \mathrm{g}$ and $1000 \mu \mathrm{g} / \mathrm{ml}$ of MCFA products in culture medium and the final volume is $200 \mu \mathrm{l}$ per well. Each concentration of MCFAs product was repeated 3-wells. The plate was then incubated in the $5 \% \mathrm{CO} 2$ incubator at $37{ }^{\circ} \mathrm{C}$ for $24 \mathrm{~h}$ for determining the cytotoxicity and cell proliferation by 3-(4,5-dimethylthiazol-2-yl)-2,5-diphenyltetrazolium bromide (MTT kit, Abcam, USA), according to the manufacturer's protocols

\section{Statistical analysis}


Statistical analysis was performed using IBM SPSS software (SPSS 23.0 for Windows; IBM, Chicago, IL, USA). A $P$-value $<0.05$ was considered to be statistically significant. Differences among the groups were tested by Duncan's multiple comparison methods.

\section{Abbreviations}

ASF, African swine fever: ASF; African swine fever virus: ASFV; Primary porcine alveolar macrophages: PAMs; Haemadsorption: HAD; Dulbecco's Modified Eagle Medium: DMEM; Tandem repeat sequences: TRS; (4,5-dimethylthiazol-2-yl)-2,5-diphenyltetrazolium bromide: MTT; World Organization for Animal Health: OIE; 50\% HAD doses: HAD50; National Center for Biotechnology Information: NCBl; Phosphatebuffered saline: PBS; Royal Agrifirm Group: RAG.

\section{Declarations}

\section{Ethics approval and consent to participate}

The study was conducted in compliance with the institutional rules for the care and use of laboratory animals and using a protocol approved by the Ministry of Agriculture and Rural Development (MARD) Vietnam (TCVN 8402:2010).

\section{Consent for publication}

Not applicable.

\section{Availability of data and material}

The dataset analyzed during the current study is available from the corresponding author on reasonable request.

\section{Competing interests}

The authors declare that they have no competing interests.

\section{Funding}

This work was supported by the Ministry of Science and Technology to Hoang Vu Dang (Project code: ĐTĐL.CN-75/19).

\section{Authors' Contributions}


HTTT, ADT, and HVD conceived and designed the experiments. HTTT, ADT, DVL, TVH, CTN, NTC, HTN, and ADT performed the experiments. HTTT, ADT, KL, GB and HVD analyzed the data. HVD and KL contributed the reagents, materials, and analytical tools. HTTT, ADT, MDV, KL, GB, and HVD wrote the manuscript. All authors read and approved the final manuscript.

\section{Acknowledgements}

The authors would like to thank the Royal Agrifirm Group for providing the funding and in-kind resources necessary to complete this project

\section{References}

1. Eustace Montgomery R. On A Form of Swine Fever Occurring in British East Africa (Kenya Colony). Journal of Comparative Pathology Therapeutics. 1921;34:159-91.

2. OIE. African swine fever. Manual of Diagnostic Tests and Vaccines for Terrestrial Animals 2012, Chap. 2812012.

3. Emergence of African Swine Fever in China Transbound Emerg Dis 2018.

Zhou X, Li N, Luo Y, Liu Y, Miao F, Chen T, Zhang S, Cao P, Li X, Tian K, et al: Emergence of African Swine Fever in China, 2018. Transbound Emerg Dis 2018.

4. Tran HTT, Dang AK, Ly DV, Vu HT, Hoang TV, Nguyen CT, Chu NT, Nguye VT, Nguyen HT, Truong AD, et al: An improvement of real-time PCR system based on probe modification is required for accurate detection of African swine fever virus in clinical samples in Vietnam. Asian-Australas J Anim Sci 2019.

5. Kim SH, Kim J, Son K, Choi Y, Jeong HS, Kim YK, Park JE, Hong YJ, Lee SI, Wang SJ, et al. Wild boar harbouring African swine fever virus in the demilitarized zone in South Korea, 2019. Emerg Microbes Infect. 2020;9(1):628-30.

6. Kim HJ, Lee MJ, Lee SK, Kim DY, Seo SJ, Kang HE, Nam HM. African Swine Fever Virus in Pork Brought into South Korea by Travelers from China, August 2018. Emerg Infect Dis. 2019;25(6):12313.

7. Sanchez EG, Perez-Nunez D, Revilla Y. Development of vaccines against African swine fever virus. Virus Res. 2019;265:150-5.

8. Gaudreault NN, Richt JA. Subunit Vaccine Approaches for African Swine Fever Virus. Vaccines 2019, $7(2)$.

9. Dixon LK, Sun H, Roberts H. African swine fever. Antiviral research. 2019;165:34-41.

10. Revilla Y, Perez-Nunez D, Richt JA. African Swine Fever Virus Biology and Vaccine Approaches. Adv Virus Res. 2018;100:41-74. 
11. Niederwerder MC, Stoian AMM, Rowland RRR, Dritz SS, Petrovan V, Constance LA, Gebhardt JT, Olcha M, Jones CK, Woodworth JC, et al. Infectious Dose of African Swine Fever Virus When Consumed Naturally in Liquid or Feed. Emerg Infect Dis. 2019;25(5):891-7.

12. Mazur-Panasiuk N, Zmudzki J, Wozniakowski G. African Swine Fever Virus - Persistence in Different Environmental Conditions and the Possibility of its Indirect Transmission. Journal of veterinary research. 2019;63(3):303-10.

13. Davies K, Goatley LC, Guinat C, Netherton CL, Gubbins S, Dixon LK, Reis AL. Survival of African Swine Fever Virus in Excretions from Pigs Experimentally Infected with the Georgia 2007/1 Isolate. Transbound Emerg Dis. 2017;64(2):425-31.

14. Eble PL, Hagenaars TJ, Weesendorp E, Quak S, Moonen-Leusen HW, Loeffen WLA. Transmission of African Swine Fever Virus via carrier (survivor) pigs does occur. Vet Microbiol. 2019;237:108345.

15. Dee S, Neill C, Clement T, Christopher-Hennings J, Nelson E. An evaluation of a liquid antimicrobial $($ Sal $C U R B(R)$ ) for reducing the risk of porcine epidemic diarrhea virus infection of naive pigs during consumption of contaminated feed. BMC Vet Res. 2014;10:220.

16. Cochrane RA, Dritz SS, Woodworth JC, Huss AR, Stark CR, Hesse RA, Zhang J, Tokach MD, Bai J, Jones CK. Evaluating Chemical Mitigation of Porcine Epidemic Diarrhea Virus (PEDV) in Swine Feed and Ingredients. Kansas Agricultural Experiment Station Research Reports 2015, 1(7).

17. Hakobyan A, Arabyan E, Kotsinyan A, Karalyan Z, Sahakyan H, Arakelov V, Nazaryan K, Ferreira F, Zakaryan $\mathrm{H}$. Inhibition of African swine fever virus infection by genkwanin. Antiviral research. 2019;167:78-82.

18. Zhang W, Qiao H, Lv Y, Wang J, Chen X, Hou Y, Tan R, Li E. Apigenin inhibits enterovirus-71 infection by disrupting viral RNA association with trans-acting factors. PLoS One. 2014;9(10):e110429.

19. Dixon LK, Islam M, Nash R, Reis AL. African swine fever virus evasion of host defences. Virus Res. 2019;266:25-33.

20. Cwynar P, Stojkov J, Wlazlak K. African Swine Fever Status in Europe. Viruses 2019, 11(4).

21. Chenais E, Depner K, Guberti V, Dietze K, Viltrop A, Stahl K. Epidemiological considerations on African swine fever in Europe 2014-2018. Porcine Health Manag. 2019;5:6.

22. Zentek J, Buchheit-Renko S, Ferrara F, Vahjen W, Van Kessel AG, Pieper R. Nutritional and physiological role of medium-chain triglycerides and medium-chain fatty acids in piglets. Animal health research reviews. 2011;12(1):83-93.

23. Li L, Wang Q, Ge S, Liu Y, Liu C, Liu F, Hu Y, Li J, Bao J, Ren W, et al: Infection of African swine fever in wild boar, China, 2018. Transbound Emerg Dis 2018.

24. Ge S, Li J, Fan X, Liu F, Li L, Wang Q, Ren W, Bao J, Liu C, Wang H, et al. Molecular Characterization of African Swine Fever Virus, China, 2018. Emerg Infect Dis. 2018;24(11):2131-3.

25. Kolbasov D, Titov I, Tsybanov S, Gogin A, Malogolovkin A. African Swine Fever Virus, Siberia, Russia, 2017. Emerg Infect Dis. 2018;24(4):796-8. 
26. Zani L, Forth JH, Forth L, Nurmoja I, Leidenberger S, Henke J, Carlson J, Breidenstein C, Viltrop A, Hoper D, et al. Deletion at the 5 -end of Estonian ASFV strains associated with an attenuated phenotype. Sci Rep. 2018;8(1):6510.

27. Sanna G, Dei Giudici S, Bacciu D, Angioi PP, Giammarioli M, De Mia GM, Oggiano A. Improved Strategy for Molecular Characterization of African Swine Fever Viruses from Sardinia, Based on Analysis of p30, CD2V and I73R/I329L Variable Regions. Transbound Emerg Dis. 2017;64(4):12806.

28. Gallardo C, Fernandez-Pinero J, Pelayo V, Gazaev I, Markowska-Daniel I, Pridotkas G, Nieto R, Fernandez-Pacheco P, Bokhan S, Nevolko O, et al. Genetic variation among African swine fever genotype II viruses, eastern and central Europe. Emerg Infect Dis. 2014;20(9):1544-7.

29. Quembo CJ, Jori F, Vosloo W, Heath L. Genetic characterization of African swine fever virus isolates from soft ticks at the wildlife/domestic interface in Mozambique and identification of a novel genotype. Transbound Emerg Dis. 2018;65(2):420-31.

30. Chapman DA, Darby AC, Da Silva M, Upton C, Radford AD, Dixon LK. Genomic analysis of highly virulent Georgia 2007/1 isolate of African swine fever virus. Emerg Infect Dis. 2011;17(4):599-605.

31. Juszkiewicz M, Walczak M, Wozniakowski G. Characteristics of Selected Active Substances used in Disinfectants and their Virucidal Activity Against ASFV. Journal of veterinary research. 2019;63(1):17-25.

32. Hakobyan A, Arabyan E, Avetisyan A, Abroyan L, Hakobyan L, Zakaryan H. Apigenin inhibits African swine fever virus infection in vitro. Arch Virol. 2016;161(12):3445-53.

33. Food Safety Authority E. Evaluation of possible mitigation measures to prevent introduction and spread of African swine fever virus through wild boar, vol. 12; 2014.

34. Guinat C, Gogin A, Blome S, Keil G, Pollin R, Pfeiffer DU, Dixon L. Transmission routes of African swine fever virus to domestic pigs: current knowledge and future research directions. Vet Rec. 2016;178(11):262-7.

35. Dierick NA, Decuypere JA, Molly K, Van Beek E, Vanderbeke E. The combined use of triacylglycerols (TAGs) containing medium chain fatty acids (MCFAs) and exogenous lipolytic enzymes as an alternative to nutritional antibiotics in piglet nutrition: II. In vivo release of MCFAs in gastric cannulated and slaughtered piglets by endogenous and exogenous lipases; effects on the luminal gut flora and growth performance. Livestock Production Science. 2002;76(1):1-16.

36. Huang CB, Alimova Y, Myers TM, Ebersole JL. Short- and medium-chain fatty acids exhibit antimicrobial activity for oral microorganisms. Arch Oral Biol. 2011;56(7):650-4.

37. Petschow BW, Batema RP, Ford LL. Susceptibility of Helicobacter pylori to bactericidal properties of medium-chain monoglycerides and free fatty acids. Antimicrob Agents Chemother. 1996;40(2):3026.

38. Vande Maele L, Heyndrickx M, Maes D, De Pauw N, Mahu M, Verlinden M, Haesebrouck F, Martel A, Pasmans F, Boyen F. In vitro susceptibility of Brachyspira hyodysenteriae to organic acids and essential oil components. J Vet Med Sci. 2016;78(2):325-8. 
39. King DP, Reid SM, Hutchings GH, Grierson SS, Wilkinson PJ, Dixon LK, Bastos AD, Drew TW. Development of a TaqMan PCR assay with internal amplification control for the detection of African swine fever virus. J Virol Methods. 2003;107(1):53-61.

40. Malmquist WA, Hay D. Hemadsorption and cytopathic effect produced by African Swine Fever virus in swine bone marrow and buffy coat cultures. Am J Vet Res. 1960;21:104-8.

41. Reed L, Muench J. H: A simple method of estimaing fifty percent endpoints. Am J Epidemiol. 1938;27:493-7.

42. Kumar S, Stecher G, Tamura K. MEGA7: Molecular Evolutionary Genetics Analysis Version 7.0 for Bigger Datasets. Mol Biol Evol. 2016;33(7):1870-4.

\section{Supplementary Figure Legend}

Additional file 1. The original of electrophoresis gel: The results of PCR detection of ASFV isolated in PAMs cell, including p72, CD2v, and TRS of ASFV genome on 1.5\% agarose gel.

\section{Figures}



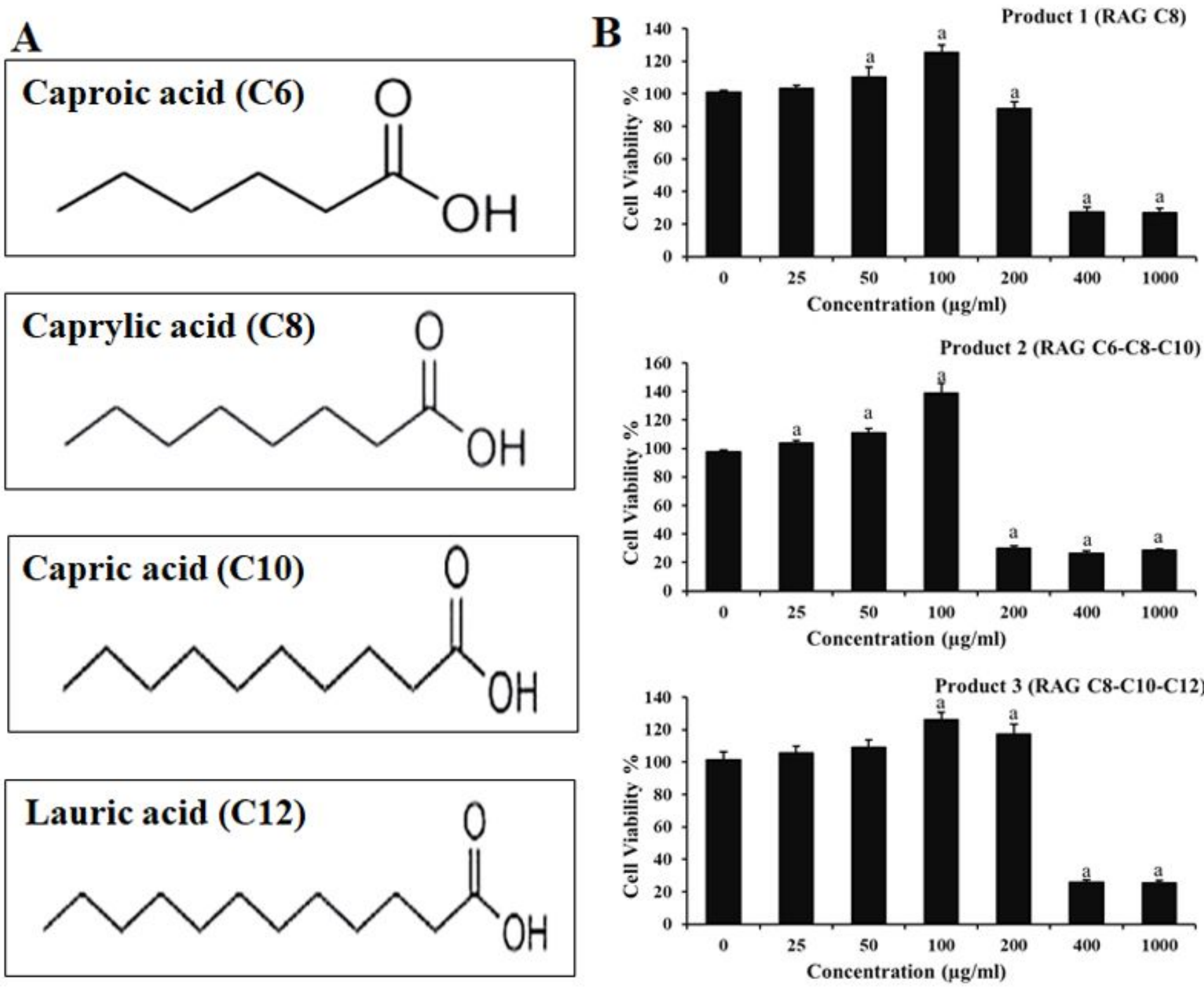

Figure 1

(A) Chemical structures of MCFA derivatives and (B) The effects of MCFAs on PAMs cell at the cell viability by MTT assay. Data are presented as the mean \pm SEM of three independent experiments indicate the significant differences between the control and treatment groups was determined by one-way ANOVA $($ a: $\mathrm{P}<0.05)$. 
A Pig/Hanoi/2019/01

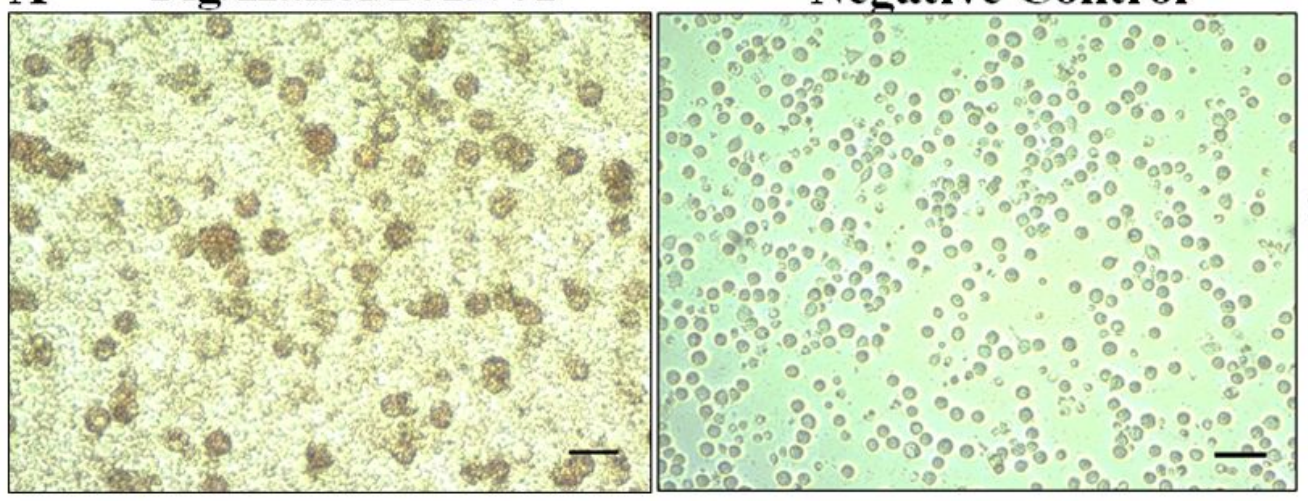

B

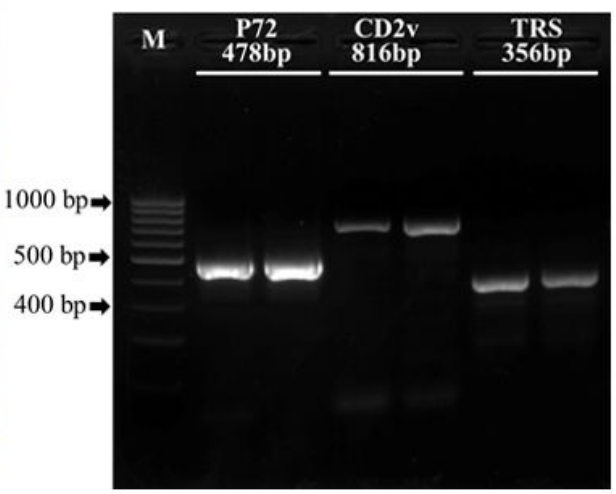

\section{Figure 2}

HAD assay and PCR verification for virus isolation. (A) HAD assay of the spleen homogenate. The 10times dilution of the supernatant of the homogenate was inoculated into PAMs cell with $1 \%$ pig red blood cells. HAD was observed for 4 days. Scale bar: $200 \mu \mathrm{m}$. (B) PCR detection of ASFV isolated in PAMs cell, including $\mathrm{p} 72, \mathrm{CD} 2 \mathrm{v}$, and TRS of ASFV genome. 

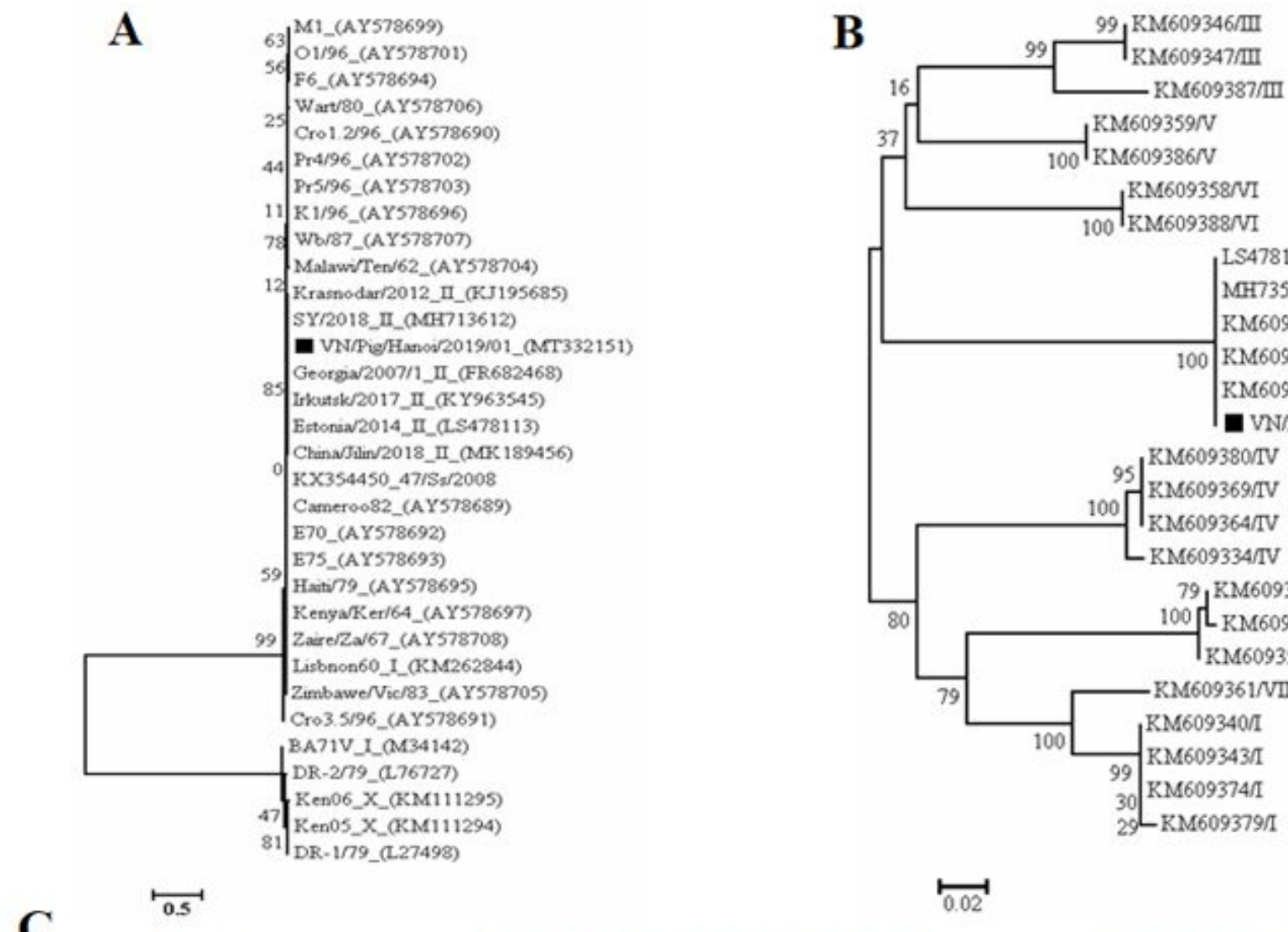

Georgia07 FR682468

SY18_MH $71 \overline{7} 104$

$\mathrm{Pig} / \overline{\mathrm{H}} \mathrm{LJ} / 2018$ MK333180

AnhuiXCGQ MK'̄ 28995

Pig/Hanoi/2019/01_MT332153

Arm07 KJ620028

Abk07-KJ620029

Che07-KJ620030

$\mathrm{Az} 08 \mathrm{D}-\mathrm{KJ} 620035$

Ukr12/Z apo KJ 620037

Belgium 20̄/1 LR536725

CN201801_MH7351̄44

\begin{abstract}
CAAGTATATAGGAATATATAGGAATATATAG
AAATATATAGAAATAGCTAAGCTTAATACTAAT CAAGTATATAGGAATATATAGGAATATATAGGAATATATAGAAATATATAGAAATAGCTAAGCTTAATACTAAT CAAGTATATAGGATATATAGGAATATATAGGAATATATAGAAATATATAGAAATAGCTAAGCTTAATACTAAT CAAGTATATAGGAATATATAGGAATATATAGGAATATATAGAAATATATAGAAATAGCTAAGCTTAATACTAAT CAAGTATATAGGAATATATAGgAATATATAGGAATATATAGAAATATATAGAAATAGCTAAGCTTAATACTAAT CAAGTATATAGGAATATATAGGAATATATAG - AAATATATAGAAATAGCTAAGCTTAATACTAAT CAAGTATATAGGAATATATAGGAATATATAG -.... AAATATATAGAAATAGC TAAGCTTAATACTAAT CAAGTATATAGGATATATAGGAATATATAG_....... AAATATATAGAAATAGCTAAGCTTAATACTAAT CAAGTATATAGGAATATATAGGATATATAG___. AAATATATAGAAATAGCTAAGCTTAATACTAAT CAAGTATATAGGAATATATAGGAATATATAGGAATATATAGAAATATATAGAAATAGCTAAGCTTAATACTAAT CAAGTATATAGGATATATAGGAATATATAGGATATATAGAAATATATAGAAATAGCTAAGCTTAATACTAAT CAAGTATATAGGAATATATAGGAATATATAGGAATATATAGAAATATATAGAAATAGCTAAGCTTAATACTAAT
\end{abstract}

\section{Figure 3}

Phylogenetic analysis of Pig/Hanoi/2019/01 based on its partial p72 (A), EP402R gene encoding the $\mathrm{CD} 2 \mathrm{v}$ protein $(\mathrm{B})$ genes. The sequences of the $\mathrm{p} 72$ and EP402R gene encoding the CD2v protein genes of representative ASF virus were downloaded from the NCBI database. The neighbor-joining method was used to construct phylogenetic trees using MEGA 7 software. Numbers along branches indicate bootstrap values $>70 \%$ (1,000 replicates). The black square indicates the ASF virus isolate from this study. Scale bars indicate nucleotide substitutions per site; (C) Alignment of the partial nucleotide sequence of the intergenic region between I73R and I329L of ASFV strain isolated from the capital Hanoi of Vietnam with reference ASFV strains. The mutation that results in the insertion of a single nucleotide internal repeat sequence (GGAATATATA) in the Pig/Hanoi/2019/01 is indicated by gray shading. 


\begin{tabular}{l|c|c|c|c}
\hline \multirow{2}{*}{\multicolumn{1}{c|}{ Groups }} & \multicolumn{4}{|c}{ Medium chain fatty acid (MCFA) in Feed (Mean \pm SE) } \\
\cline { 2 - 5 } & $\mathbf{0 . 1 2 5} \%$ & $\mathbf{0 . 2 5} \%$ & $\mathbf{0 . 3 7 5} \%$ & $\mathbf{0 . 5 0} \%$ \\
\hline Positive control & $25.85 \pm 0.67$ & $26.52 \pm 0.82$ & $26.4 \pm 0.69$ & $26.09 \pm 0.19$ \\
\hline Product 1 (RAG C8) & $27.55 \pm 0.71$ & $28.1 \pm 1.16$ & $29.44 \pm 0.47$ & $30.02 \pm 0.98$ \\
\hline Product 2 & & & & \\
(RAG C6-C8-C10) & $29.34 \pm 0.51$ & $28.80 \pm 0.92$ & $30.68 \pm 0.79$ & $30.80 \pm 0.91$ \\
\hline Product 3 & & & & \\
(RAG C8-C10-C12) & $26.94 \pm 0.68$ & $29.29 \pm 1.37$ & $29.58 \pm 0.43$ & $30.95 \pm 1.43$ \\
\hline Negative control & No Cq & No Cq & No Cq & No Cq \\
\hline
\end{tabular}
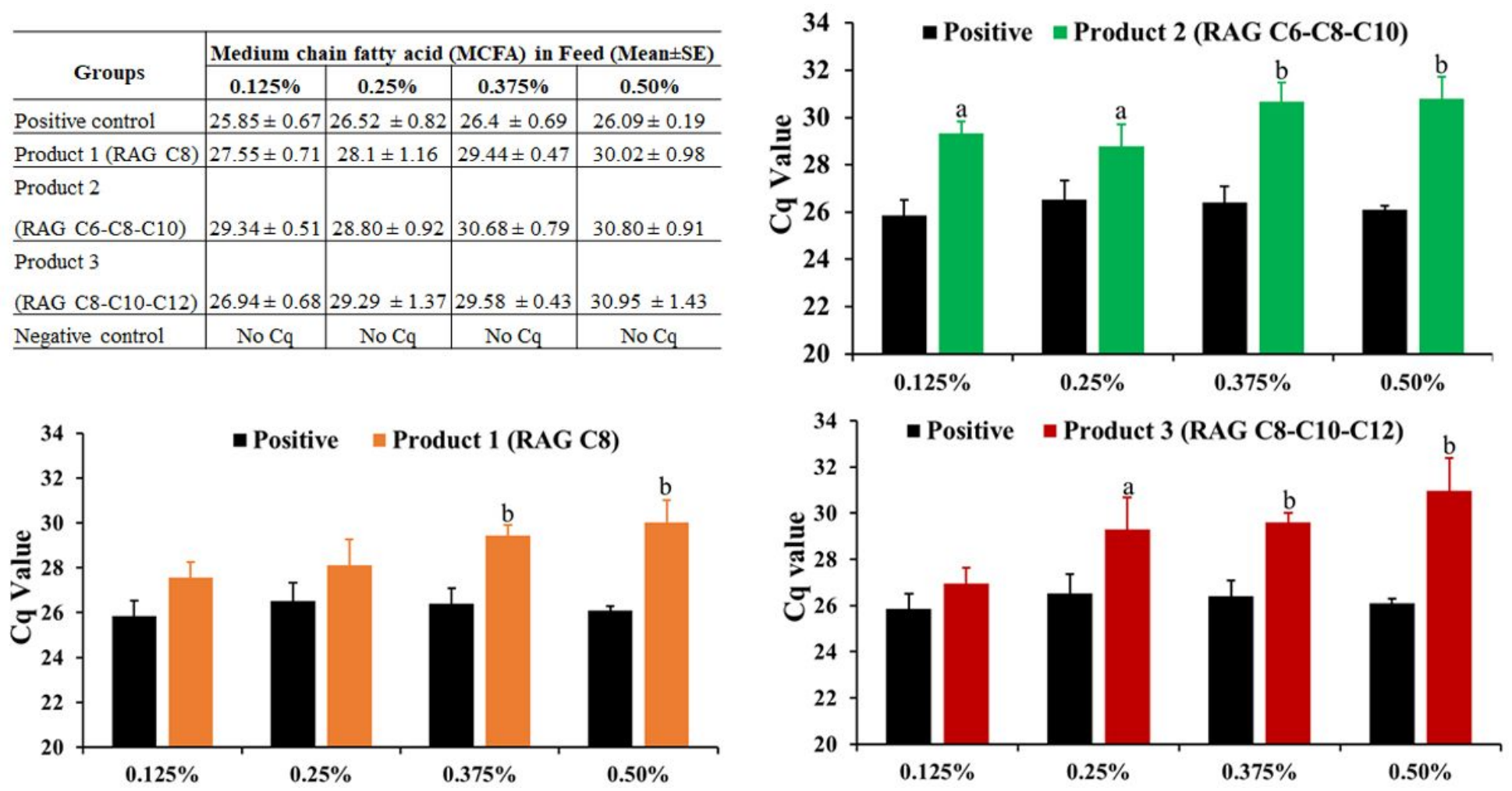

Figure 4

MCFA pre-treated feed in exposure to $1 \times 105 \mathrm{HAD} 50 / \mathrm{g}$ of $\mathrm{Pig} / \mathrm{Hanoi} / 2019 / 01$ strain after 24 -hour postinoculation. The viral DNA of the ASF virus in supernatants collected from feed exposed and then, realtime PCR amplification of the p72 gene was performed to detect the presence of viral DNA in MCFAs treated feeds. Data are presented as the mean \pm SEM of three independent experiments: a: $P<0.05$ and b: $P<0.01$. 


\begin{tabular}{l|c|c|c|c}
\hline \multirow{2}{*}{\multicolumn{1}{c|}{ Groups }} & \multicolumn{4}{|c}{ Medium chain fatty acid (MCFA) in Feed (Mean \pm SE) } \\
\cline { 2 - 5 } & $\mathbf{0 . 1 2 5} \%$ & $\mathbf{0 . 2 5} \%$ & $\mathbf{0 . 3 7 5} \%$ & $\mathbf{0 . 5 0 \%}$ \\
\hline Positive control & $35.13 \pm 0.11$ & $34.98 \pm 0.10$ & $34.65 \pm 0.37$ & $35.04 \pm 0.28$ \\
\hline Product 1 (RAG C8) & $35.38 \pm 0.24$ & $35.58 \pm 0.68$ & $37.95 \pm 0.53$ & $38.38 \pm 0.44$ \\
\hline Product 2 & & & & \\
(RAG C6-C8-C10) & $35.25 \pm 0.11$ & $35.20 \pm 0.10$ & $36.16 \pm 0.32$ & $37.09 \pm 0.81$ \\
\hline Product 3 & & & & \\
(RAG C8-C10-C12) & $35.49 \pm 0.51$ & $35.69 \pm 0.45$ & $37.22 \pm 0.25$ & $38.33 \pm 0.58$ \\
\hline Negative control & No Cq & No Cq & No Cq & No Cq \\
\hline
\end{tabular}
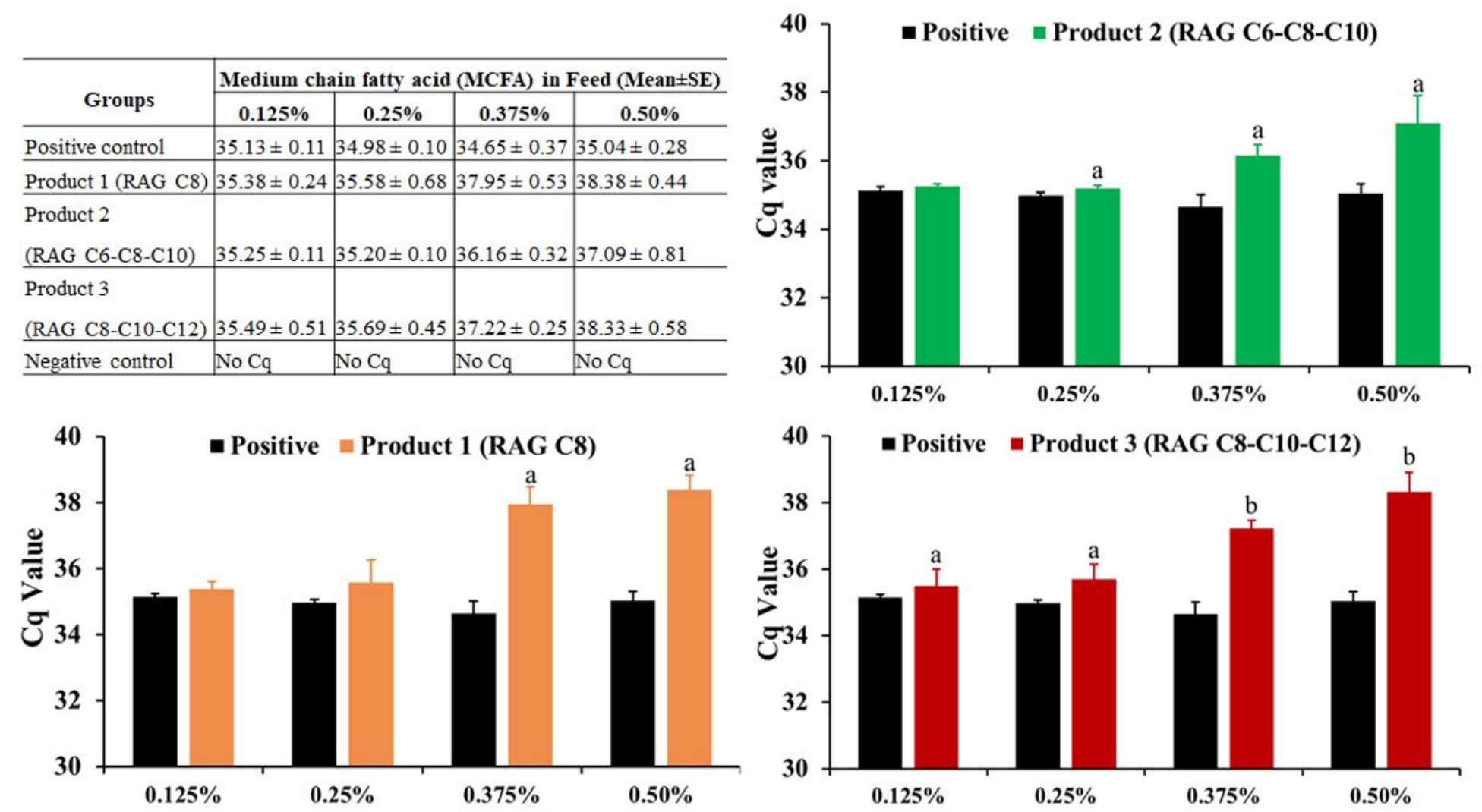

\section{Figure 5}

MCFA pre-treated water in exposure to $1 \times 101 \mathrm{HAD} 50 / \mathrm{ml}$ of Pig/Hanoi/2019/01 strain after 24-hour postinoculation. The viral DNA of the ASF virus collected from water exposure and then, real-time PCR amplification of the p72 gene was performed to detect the presence of viral DNA in MCFAs treated waters. Data are presented as the mean \pm SEM of three independent experiments: $a: P<0.05$ and $b$ : $P<$ 0.01 .

\section{Supplementary Files}

This is a list of supplementary files associated with this preprint. Click to download.

- SupplementFig.S1.tif 